\title{
Tetrahedral approximation in normal coordinate calculations of high polymers
}

\author{
(Received 10 December 1964)
}

IN MAKING normal vibration calculations for polymer molecules it is common practice to approximate near-tetrahedral CCC angles by tetrahedral angles [1-6]. While it has been noted [4] that this is an approximation, no estimate has been made of the possible magnitude of the errors inherent in its use. Since the CCC angle in polymers can be significantly different from tetrahedral $\left(112^{\circ}\right.$ in polyethylene [7] and paraffins [8], $114^{\circ}$ in polypropylene [9], $116^{\circ}$ in polystyrene $[10])$, we have investigated the effect of using non-tetrahedral angles in the calculation of frequencies and normal modes.

As an example we have computed the frequencies of a polyethylene chain in which the planar zig-zag bond angle is $112^{\circ}$ and the $\mathrm{HCH}$ angle is $107^{\circ}$, in conformity with values found in exact crystal structure determinations of long-chain $n$-paraffins [8]. The force field was the valence force field of SchaCHTSCHNEIDER and SNYDER [4], and their set III force constants were used. The results are shown in Table 1. No significant difference was found in the potential energy distributions for the corresponding frequencies in Table 1, although it will be seen that some large frequency shifts occur, as well as two instances in which the order of frequencies is inverted.

Table 1. Calculated frequencies of polyethylene

\begin{tabular}{cc}
\hline All angles tetrahedral & $\mathrm{CCC}=112^{\circ}, \mathrm{HCH}=107^{\circ}$ \\
\hline 2930 & 2924 \\
2912 & 2908 \\
2864 & 2870 \\
2851 & 2855 \\
1474 & 1452 \\
1444 & 1422 \\
1412 & 1433 \\
1295 & 1315 \\
1172 & 1140 \\
1167 & 1184 \\
1132 & 1132 \\
1064 & 1080 \\
1062 & 1078 \\
720 & 688 \\
\hline
\end{tabular}

There are two main reasons for the frequency shifts observed in Table 1 . The first is that the change in geometry has resulted in a change in $G$ matrix elements. In some instances this was large: for example, $G_{R \gamma^{\prime}}$ changed by $6.4 \%, G_{R R^{\prime}}$ by $12.0 \%, G_{\delta \gamma}$ by $-5 \cdot 7 \%$. (The symbols refer to the following internal coordinates: $R-C C$ stretch, $\gamma-C C H$ bend, $\delta-H C H$ bend.) Such changes would be expected to occur in the calculations for other polymers and will in fact probably be larger because of the greater departure from tetrahedral geometry in these cases. The second reason for such frequency shifts is that the force constants, which have been refined from

[1] H. Tadokoro, J. Chem. Phys. 33, 1558 (1960).

[2] T. Miyazawa, J. Chem. Phys. 35, 693 (1961).

[3] M. Tasumi, T. Shimanoudir and T. Miyazawa, J. Mol. Spectrosc. 9, 261 (1962).

[4] J. H. Sohachischneider and R. G. SNYzie, Spectrochim. Acta 19, 117 (1963).

[5] T. Mryazawa, Y. Ineguchi and K. Fukushima, J. Chem. Phys. 38, 2709 (1963).

[6] R. G. SNYder and J. H. Schachtschneider, Spectrochim. Acta 20, 853 (1964).

[7] C. W. Bunn, Trans. Faraday Soc. 35, 482 (1939).

[8] P. W. Teare, Acta Cryst. 12, 294 (1959).

[9] G. NatTa and P. Corradini, Nuovo Cimento Suppl. 15, 40 (1960).

[10] G. Natta, P. Corradini and I. W. Bassi, Nuovo Cimento Suppl. 15, 68 (1960). 
a series of $n$-paraffins of assumed tetrahedral geometry, are really not transferable to the nontetrahedral structures since these constants were defined for a particular set of redundant co. ordinates [11]. It is difficult to evaluate the relative importance of these two factors, but it can be said that frequency shifts of the observed order could easily result from the differences in $G$ matrix elements which have been obtained.

A more serious consequence of assuming tetrahedral geometry is present in those calculations $[1,2,3,5]$ where, rather than solely transferring force constants from smaller molecules $[4,6]$, the force field has been refined to give the best fit to the observed polymer frequencies. In this case it is possible that the force constants obtained for tetrahedral geometry could in sume cases be significantly different from those which would be obtained for non-tetrahedral geometry. As an illustration, we have calculated the bond stretching constant, $K_{R}$, and the angle bending constant, $H_{\phi}$, for a polyethylene chain in which the $C H_{2}$ groups are taken as point masses. A fit was made to assumed frequencies of $1065 \mathrm{~cm}^{-1}$ for the in-phase chain vibration and $1135 \mathrm{~cm}^{-1}$ for the out-of-phase vibration [12]. For a tetrahedral CCC angle: $K_{R}=4.01 \mathrm{mdyn} / \AA, H_{\phi}=$ $0.89 \mathrm{mdyn} \AA$; for a $\mathrm{CCC}$ angle of $112^{\circ}: K_{R}=3.89 \mathrm{mdyn} / \AA, H_{\phi}=0.97 \mathrm{mdyn} / \AA$. Thus the use of tetrahedral geometry may not be reflected in any poorer frequency fit, but the difference in force constants could significantly alter the predicted normal coordinates. It is clear that caution must at present be maintained concerning the details of normal coordinates for high polymers.

Acknowledgement-This research was supported by a National Science Foundation grant.

Harrison M. Randall Laboratory of Physics

C. G. Opaskar

University of Michigan

S. KRIMM

Ann Arbor

Michigan

[11] R. G. SNyder, Private communication.

[12] S. KRIMM, Fortschr. Hochpolym.-Forsch. 2, 51 (1960). 